ANNALES

POLONICI MATHEMATICI

LXXVI.3 (2001)

\title{
Asymptotic stability of a system of randomly connected transformations on Polish spaces
}

\author{
by KatARZyna HorbaCz (Katowice)
}

\begin{abstract}
We give sufficient conditions for the existence of a matrix of probabilities $\left[p_{i k}\right]_{i, k=1}^{N}$ such that a system of randomly chosen transformations $\Pi_{k}, k=1, \ldots, N$, with probabilities $p_{i k}$ is asymptotically stable.
\end{abstract}

0. Introduction. We consider a system of randomly connected transformations on a Polish space $Y$ (see K. Horbacz [2]).

Let $Y$ be a Polish space, i.e. a separable, complete metric space, which is the phase space of some dynamical system. In the deterministic case the dynamics can be described in terms of a function $\Pi: \mathbb{R}_{+} \times Y \rightarrow Y$ so that a point starting from $x_{0} \in Y$ at time $t_{0}$ is in position $\Pi\left(t-t_{0}, x_{0}\right)$ at time $t>t_{0}$.

In this paper we consider a random dynamics. We assume that a point can move according to one of the transformations $\Pi_{k}: \mathbb{R}_{+} \times Y \rightarrow Y$ from a set $\left\{\Pi_{1}, \ldots, \Pi_{N}\right\}$. The choice of the initial transformations is random and changes randomly at random moments $t_{k}$. This system is called a system of randomly connected transformations. The probabilities determining the frequency with which the maps $\Pi_{k}$ can be chosen are described by means of a stochastic matrix $\left[p_{i k}(x)\right]_{i, k=1}^{N}$. We give sufficient conditions for the existence of a stochastic matrix as above such that the system $\Pi_{k}, k=1, \ldots, N$, with probabilities $p_{i k}$ is asymptotically stable.

It should be underlined that our stability criterion is valid in a general class of metric spaces (Polish spaces) which are not necessarily locally compact. Thus these results are applicable to infinite-dimensional systems.

In the case when the transformation $\Pi_{k}$ does not depend on the variable $t$ and $p_{i j}=p_{i}$ for $j=1, \ldots, N$, we obtain an Iterated Function System with probabilities. In [4] A. Lasota and J. Myjak gave sufficient conditions for the existence of probabilities $\left\{p_{i}: i \in I\right\}, p_{i}: Y \rightarrow(0,1]$, such that an Iterated

2000 Mathematics Subject Classification: Primary 47A35; Secondary 58F30.

Key words and phrases: dynamical systems, Markov operator, asymptotic stability. 
Function System on measures is asymptotically stable. Our method is based on their ideas.

The organization of the paper is as follows. Section 1 contains some notation and definitions from the theory of Markov operators. In Section 2 we specify the problem to be considered. Sufficient conditions for the existence of $\left[p_{i k}\right]_{i, k=1}^{N}$ such that the corresponding system of randomly connected trasformations is asymptotically stable are given in Section 3.

1. Preliminaries. Let $(Y, \varrho)$ be a Polish space. Throughout this paper, $K(x, r)$ stands for the closed ball in $Y$ with center at $x$ and radius $r$.

We denote by $\mathcal{C}_{\varepsilon}(Y), \varepsilon>0$, the family of all sets $C \subset Y$ for which there exists a finite set $\left\{x_{1}, \ldots, x_{n}\right\} \subset Y$ such that

$$
C=\bigcup_{i=1}^{n} K\left(x_{i}, \varepsilon\right) .
$$

We denote by $\mathcal{B}(Y)$ the $\sigma$-algebra of all Borel subsets of $Y$ and by $\mathcal{M}(Y)$ the family of all finite Borel measures (nonnegative, $\sigma$-additive) on $Y$. By $\mathcal{M}_{1}(Y)$ we denote the subset of $\mathcal{M}(Y)$ such that $\mu(Y)=1$ for $\mu \in \mathcal{M}_{1}(Y)$. The elements of $\mathcal{M}_{1}(Y)$ will be called distributions. Further

$$
\mathcal{M}_{\text {sig }}(Y)=\left\{\mu_{1}-\mu_{2}: \mu_{1}, \mu_{2} \in \mathcal{M}(Y)\right\}
$$

is the space of all finite signed measures.

Let $\Theta \subset \mathcal{M}_{1}(Y)$. We call $\Theta$ tight if for every $\varepsilon>0$ there exists a compact set $K \subset Y$ such that $\mu(K) \geq 1-\varepsilon$ for all $\mu \in \Theta$.

As usual, $B(Y)$ denotes the space of all bounded Borel measurable functions $f: Y \rightarrow \mathbb{R}$, and $C(Y)$ the subspace of all bounded continuous functions. Both spaces are considered with the supremum norm $\|\cdot\|_{0}$.

For $f \in B(Y)$ and $\mu \in \mathcal{M}_{\text {sig }}(Y)$ we write

$$
\langle f, \mu\rangle=\int_{Y} f(x) \mu(d x) .
$$

We say that a sequence $\left\{\mu_{n}\right\}_{n \geq 1}, \mu_{n} \in \mathcal{M}_{1}(Y)$, converges weakly to a measure $\mu \in \mathcal{M}_{1}(Y)$ if

$$
\lim _{n \rightarrow \infty}\left\langle f, \mu_{n}\right\rangle=\langle f, \mu\rangle \quad \text { for } f \in C(Y) .
$$

In the space $\mathcal{M}_{\text {sig }}(Y)$ we introduce the Fortet-Mourier norm (see $[1,5]$ ) by setting

$$
\|\mu\|_{\mathcal{F}}=\sup \{\langle f, \mu\rangle: f \in \mathcal{F}\}
$$

where

$$
\mathcal{F}=\left\{f \in C(Y):\|f\|_{0} \leq 1 \text { and }|f(x)-f(y)| \leq \varrho(x, y) \text { for } x, y \in Y\right\} .
$$


The space $\mathcal{M}_{1}(Y)$ with the distance $\left\|\mu_{1}-\mu_{2}\right\|_{\mathcal{F}}$ is a complete metric space and the convergence

$$
\lim _{n \rightarrow \infty}\left\|\mu_{n}-\mu\right\|_{\mathcal{F}}=0 \quad \text { for } \mu_{n}, \mu \in \mathcal{M}_{1}(Y)
$$

is equivalent to the weak convergence of $\left\{\mu_{n}\right\}_{n \geq 1}$ to $\mu$.

For $A \subset Y$ we denote by $\operatorname{diam}_{\varrho} A$ the diameter of the set $A$, i.e. $\operatorname{diam}_{\varrho} A=$ $\sup \{\varrho(x, y): x, y \in A\}$.

A linear mapping $P: \mathcal{M}_{\text {sig }}(Y) \rightarrow \mathcal{M}_{\text {sig }}(Y)$ is called a Markov operator if $P\left(\mathcal{M}_{1}(Y)\right) \subset \mathcal{M}_{1}(Y)$. A measure $\mu_{*} \in \mathcal{M}(Y)$ is called invariant or stationary for $P$ if $P \mu_{*}=\mu_{*}$. A stationary probability measure is called a stationary distribution.

We define

$$
\begin{aligned}
\omega(\mu) & =\left\{\nu \in \mathcal{M}_{1}(Y): \exists_{\left\{n_{k}\right\}_{k \geq 1}}, n_{k} \rightarrow \infty, P^{n_{k}} \mu \rightarrow \nu\right\}, \\
\Gamma & =\bigcup_{\mu \in \mathcal{M}_{1}(Y)} \omega(\mu) .
\end{aligned}
$$

A Markov operator $P: \mathcal{M}_{\text {sig }}(Y) \rightarrow \mathcal{M}_{\text {sig }}(Y)$ is called a Feller operator if there is an operator $U: B(Y) \rightarrow B(Y)$ (dual to $P$ ) such that

$$
\langle U f, \mu\rangle=\langle f, P \mu\rangle \quad \text { for } f \in B(Y), \mu \in \mathcal{M}_{\text {sig }}(Y)
$$

and

$$
U f \in C(Y) \quad \text { for } f \in C(Y) \text {. }
$$

Setting $\mu=\delta_{x}$ in (1.1) we obtain

$$
U f(x)=\left\langle f, P \delta_{x}\right\rangle \quad \text { for } f \in B(Y), x \in Y,
$$

where $\delta_{x} \in \mathcal{M}_{1}(Y)$ is the point (Dirac) measure supported at $x$.

A Markov operator $P: \mathcal{M}_{\text {sig }}(Y) \rightarrow \mathcal{M}_{\text {sig }}(Y)$ is called nonexpansive if

$$
\left\|P \mu_{1}-P \mu_{2}\right\|_{\mathcal{F}} \leq\left\|\mu_{1}-\mu_{2}\right\|_{\mathcal{F}} \quad \text { for } \mu_{1}, \mu_{2} \in \mathcal{M}_{1}(Y)
$$

semi-concentrating if for every $\varepsilon>0$ there exist $C \in \mathcal{C}_{\varepsilon}$ and $\alpha>0$ such that

$$
\liminf _{n \rightarrow \infty} P^{n} \mu(C)>\alpha \quad \text { for } \mu \in \mathcal{M}_{1}(Y) ;
$$

and asymptotically stable if there exists a stationary measure $\mu_{*} \in \mathcal{M}_{1}(Y)$ such that

$$
\lim _{n \rightarrow \infty}\left\|P^{n} \mu-\mu_{*}\right\|_{\mathcal{F}}=0 \quad \text { for } \mu \in \mathcal{M}_{1}(Y) .
$$

2. Formulation of the problem. Let $(Y, \varrho)$ be a Polish space. Suppose we are given a sequence of continuous transformations $\Pi_{k}: \mathbb{R}_{+} \times Y \rightarrow$ $Y, k=1, \ldots, N$, and a sequence of random variables $\left\{t_{n}\right\}_{n \geq 1}$ such that the increments

$$
\Delta t_{1}=t_{1}-t_{0}, \ldots, \Delta t_{n}=t_{n}-t_{n-1}, \ldots \quad\left(t_{0}=0\right)
$$


are independent and have the same density distribution function $g(t)=$ $a e^{-a t}$.

Assume moreover that Borel measurable functions $p_{i}, p_{i k}: Y \rightarrow \mathbb{R}_{+}$ satisfy

$$
p_{i}(x) \geq 0, \quad \sum_{i=1}^{N} p_{i}(x)=1 \quad \text { for } x \in Y
$$

and

$$
p_{i j}(x) \geq 0, \quad \sum_{j=1}^{N} p_{i j}(x)=1 \quad \text { for } x \in Y \text { and } i, j=1, \ldots, N .
$$

The action of randomly chosen transformations can be roughly described as follows. We choose an initial point $x_{0} \in Y$. Next we randomly select an integer $k_{1}$ from $\{1, \ldots, N\}$ with some probability $p_{k_{1}}\left(x_{0}\right)$. We define

$$
x_{1}=\Pi_{k_{1}}\left(t_{1}, x_{0}\right) .
$$

Next, we select $k_{2}$ with probability $p_{k_{1} k_{2}}\left(x_{1}\right)$ and define

$$
x_{2}=\Pi_{k_{2}}\left(t_{2}-t_{1}, x_{1}\right)
$$

and so on. Thus

$$
x_{n}=\Pi_{s}\left(t_{n}-t_{n-1}, x_{n-1}\right)
$$

with probability $p_{k s}\left(x_{n-1}\right)$ if $x_{n-1}=\Pi_{k}\left(t_{n-1}-t_{n-2}, x_{n-2}\right)$.

The system of randomly chosen $\Pi_{k}$ with probabilities $p_{i k}$ is denoted by $[\Pi, p]$.

Denote by $\mu_{n}, n=0,1, \ldots$, the distribution of $x_{n}$, i.e.

$$
\mu_{n}(A)=\operatorname{prob}\left(x_{n} \in A\right) \quad \text { for } A \in \mathcal{B}(Y), n=0,1, \ldots
$$

We will give sufficient conditions for the existence of a matrix of probabilities $\left[p_{i k}\right]_{i, k=1}^{N}, p_{i k}: Y \rightarrow(0,1]$, such that the sequence $\left\{\mu_{n}\right\}_{n \geq 1}$ is weakly convergent to a unique measure $\mu_{*}$.

We change the space $Y$ in order to be able to describe the evolution of measures under some Markov operator.

Let $\bar{Y}=Y \times\{1, \ldots, N\}$ with the metric $\bar{\varrho}$ given by

$$
\bar{\varrho}((x, i),(y, j))=\varrho(x, y)+\varrho_{0}(i, j) \quad \text { for } x, y \in Y, i, j \in\{1, \ldots, N\},
$$

where $\varrho_{0}$ is some metric in $\{1, \ldots, N\}$.

We define a new sequence of transformations

$$
\bar{\Pi}_{k}: \mathbb{R}_{+} \times \bar{Y} \rightarrow \bar{Y} \quad \text { for } k=1, \ldots, N
$$

by

$$
\bar{\Pi}_{k}(t,(x, s))=\left(\Pi_{k}(t, x), k\right) .
$$


Now, for an initial point $x_{0}$ we randomly select an integer $k$ with probability $p_{k}\left(x_{0}\right)$ and we define $x_{1}=\Pi_{k}\left(t_{1}, x_{0}\right)$. Next we randomly select $s \in\{1, \ldots, N\}$ with probability $p_{k s}\left(x_{1}\right)$, and we define

$$
\left(x_{2}, s\right)=\bar{\Pi}_{s}\left(t_{2}-t_{1},\left(x_{1}, k\right)\right)
$$

and so on. Hence

$$
\left(x_{n}, s\right)=\bar{\Pi}_{s}\left(\Delta t_{n},\left(x_{n-1}, k\right)\right), \quad n=2,3, \ldots,
$$

with probability $p_{k s}\left(x_{n-1}\right)$.

The evolution of the distributions $\bar{\mu}_{n}$ on the space $\bar{Y}$, where

$$
\bar{\mu}_{n}(A \times\{s\})=\operatorname{prob}\left(x_{n} \in A \text { and } x_{n}=\Pi_{s}\left(\Delta t_{n}, x_{n-1}\right)\right), \quad n=1,2, \ldots,
$$

can be described by a Feller operator $P$, i.e. $\bar{\mu}_{n+1}=P \bar{\mu}_{n}$. It is called the transition operator for this system. To find the explicit form of $P$, we look for the dual operator $U$. A straightforward calculation shows that

$$
\begin{aligned}
U f(x, k) & =\sum_{s=1}^{N} \int_{0}^{\infty} f\left(\bar{\Pi}_{s}(t,(x, k))\right) a e^{-a t} p_{k s}(x) d t \\
& =\sum_{s=1}^{N} \int_{0}^{\infty} f\left(\Pi_{s}(t, x), s\right) a e^{-a t} p_{k s}(x) d t \quad \text { for } f \in B(\bar{Y}) .
\end{aligned}
$$

Thus (see [3]), we may find $P$ by the formula

$$
P \mu(A)=\left\langle\mathbf{1}_{A}, P \mu\right\rangle=\left\langle U \mathbf{1}_{A}, \mu\right\rangle .
$$

This gives

$$
P \mu(A)=\sum_{s=1}^{N} \int_{\bar{Y}} \int_{0}^{\infty} \mathbf{1}_{A}\left(\bar{\Pi}_{s}(t,(x, k))\right) a e^{-a t} d t p_{k s}(x) d \mu(x, k)
$$

for $\mu \in \mathcal{M}(\bar{Y})$ and $A \in \mathcal{B}(\bar{Y})$.

The weak convergence of the sequence $\left\{\mu_{n}\right\}_{n \geq 1}$ will follow from the asymptotic stability of the operator $P$.

To prove the latter we need the following three lemmas. The first was proved by T. Szarek [6].

Lemma 2.1. Let $P: \mathcal{M}_{\text {sig }}(\bar{Y}) \rightarrow \mathcal{M}_{\text {sig }}(\bar{Y})$ be a nonexpansive semiconcentrating Markov operator. Then

(i) $P$ has an invariant distribution,

(ii) $\omega(\mu) \neq \emptyset$ for every $\mu \in \mathcal{M}_{1}(\bar{Y})$,

(iii) $\Gamma=\bigcup_{\mu \in \mathcal{M}_{1}(Y)} \omega(\mu)$ is tight.

In [5] A. Lasota and J. A. Yorke proved the following result.

Lemma 2.2. Let $P$ be a nonexpansive Markov operator. Assume that for every $\varepsilon>0$ there is a $\Delta>0$ with the following property: for every 
$\mu_{1}, \mu_{2} \in \mathcal{M}_{1}(\bar{Y})$ there exists a Borel measurable set $A$ with $\operatorname{diam}_{\bar{\varrho}}(A) \leq \varepsilon$ and an integer $n_{0}$ such that

$$
P^{n_{0}} \mu_{i}(A) \geq \Delta \quad \text { for } i=1,2 .
$$

Then $P$ satisfies

$$
\lim _{n \rightarrow \infty}\left\|P^{n} \mu_{1}-P^{n} \mu_{2}\right\|_{\mathcal{F}}=0 \quad \text { for } \mu_{1}, \mu_{2} \in \mathcal{M}_{1}(\bar{Y}) .
$$

We also need the following elementary lemma whose proof is left to the reader.

Lemma 2.3. Let $q: \mathbb{R}_{+} \rightarrow(\delta, \infty), \delta \geq 0$, be a nonincreasing function and let $\varepsilon>0$. Then there exists a nonincreasing function $\bar{q}: \mathbb{R}_{+} \rightarrow(\delta, \delta+\varepsilon)$ such that

$$
\delta<\bar{q}(t)<q(t) \quad \text { and } \quad|\bar{q}(t)-\bar{q}(s)| \leq \varepsilon|t-s| \quad \text { for } t, s \geq 0 .
$$

3. Main result. We now formulate the main result of this paper.

TheOREm 3.1. Assume that the sequence of transformations $\Pi_{k}: \mathbb{R}_{+} \times Y$ $\rightarrow Y$ satisfies

$$
\varrho\left(\Pi_{k}(t, x), \Pi_{k}(t, y)\right) \leq L_{k} e^{-\lambda t} \varrho(x, y)
$$

for $x, y \in A, t \geq 0$ and $k=1, \ldots, N$, on every bounded set $A \subset Y$. Assume moreover that the positive constants $a, \lambda$ and $L_{1}$ satisfy

$$
L_{1}-\lambda / a<1 .
$$

If in addition there is a point $x_{*} \in Y$ such that

$$
\sup \left\{\varrho\left(\Pi_{k}\left(t, x_{*}\right), x_{*}\right): t \geq 0\right\}<\infty \quad \text { for } k=1, \ldots, N,
$$

then there exists a matrix of probabilities $p_{i k}: Y \rightarrow(0,1]$ satisfying

$$
\sum_{k=1}^{N} p_{i k}(x)=1
$$

for $x \in Y$ and $i=1, \ldots, N$ such that the sequence $\left\{\mu_{n}\right\}_{n \geq 1}$ defined by (2.2) is weakly convergent to a distribution $\mu_{*}$.

Proof. For $x \in Y$ set

$$
|x|_{*}=\varrho\left(x, x_{*}\right) .
$$

Let $0<\delta<\left(1+\lambda / a-L_{1}\right) / N$. For $i \in\{2, \ldots, N\}$ and $r \geq 0$ define

$$
\begin{aligned}
& \sigma_{i}(r)=\sup \left\{\varrho\left(\Pi_{i}(t, x), x_{*}\right):|x|_{*} \leq r, t \geq 0\right\}, \\
& r_{i}(r)=\sup \left\{\frac{\varrho\left(\Pi_{i}(t, x), \Pi_{i}(t, y)\right)}{e^{-\lambda t} \varrho(x, y)}:|x|_{*},|y|_{*} \leq r, x \neq y \text { and } t \geq 0\right\},
\end{aligned}
$$




$$
q_{i}(r)=\min \left\{\frac{\delta}{1+r_{i}(r)}, \frac{\delta}{1+\sigma_{i}(r)}\right\}, \quad \sup \emptyset=0 .
$$

Fix $\varepsilon>0$ such that $\delta+\varepsilon<1$. Using Lemma 2.3 for $\varepsilon$, choose a sequence of functions $\bar{q}_{i}, i=2, \ldots, N$. Define

$$
\begin{array}{ll}
p_{i 1}(x)=1-\sum_{s=2}^{N} \bar{q}_{s}\left(|x|_{*}\right) & \text { for } i=1, \ldots, N \\
p_{i k}(x)=\bar{q}_{k}\left(|x|_{*}\right) & \text { for } i=1, \ldots, N \text { and } k=2, \ldots, N .
\end{array}
$$

Consider now the resulting system $[\Pi, p]$ and let $P$ and $U$ be given by (2.4) and (2.3), respectively.

Claim I. There exists a metric $\varrho_{K}$ on $\bar{Y}$ such that $P$ is nonexpansive with respect to $\varrho_{K}$.

Set

$$
K=\frac{2(N-1) N \varepsilon}{1-L_{1} a /(a+\lambda)} .
$$

Define

$$
\varrho_{K}((x, i),(y, j))=K\left(\varrho(x, y)+\varrho_{0}(i, j)\right)
$$

for $x, y \in Y$ and $i, j \in\{1, \ldots, N\}$, where

$$
\varrho_{0}(i, j)=\left\{\begin{array}{l}
c \text { for } i \neq j, \\
0 \text { for } i=j,
\end{array}\right.
$$

for $c$ such that $c K \geq 2$. Denote by $\|\cdot\|_{K}$ the Fortet-Mourier norm in $\mathcal{M}_{1}(\bar{Y})$ given by $\left\|\mu_{1}-\mu_{2}\right\|_{K}=\sup \left\{\left|\left\langle f, \mu_{1}-\mu_{2}\right\rangle\right|: f \in \mathcal{F}_{K}\right\}$, where $\mathcal{F}_{K}$ is the set of functions $f$ such that $\|f\|_{0} \leq 1$ and

$$
|f(x, i)-f(y, j)| \leq \varrho_{K}((x, i),(y, j))
$$

for $x, y \in Y, i, j \in\{1, \ldots, N\}$. To prove the nonexpansiveness it is sufficient to show that $U\left(\mathcal{F}_{K}\right) \subset \mathcal{F}_{K}$. Fix an $f \in \mathcal{F}_{K}$. Evidently $\|U f\|_{0} \leq 1$, so we have to prove that

$$
|U f(x, i)-U f(y, j)| \leq K \bar{\varrho}((x, i),(y, j))
$$

for $x, y \in Y$ and $i, j \in\{1, \ldots, N\}$. Since $\varrho_{0}(i, j)=c$ for $i \neq j$ and $K c \geq 2$, the condition (3.4) is satisfied for $i \neq j$. For $i=j$ we have

$$
\begin{aligned}
|U f(x, i)-U f(y, i)| & \leq \sum_{k=1}^{N} \int_{0}^{\infty}\left|f\left(\Pi_{k}(t, x), k\right)\right| a e^{-a t}\left|p_{i k}(x)-p_{i k}(y)\right| d t \\
& +\sum_{k=1}^{N} \int_{0}^{\infty}\left|f\left(\Pi_{k}(t, x), k\right)-f\left(\Pi_{k}(t, y), k\right)\right| p_{i k}(y) a e^{-a t} d t .
\end{aligned}
$$


Since $f \in \mathcal{F}_{K}$, we obtain

$$
\begin{aligned}
& |U f(x, i)-U f(y, i)| \\
& \leq \sum_{k=1}^{N}\left|p_{i k}(x)-p_{i k}(y)\right|+\sum_{k=1}^{N} \int_{0}^{\infty} K \varrho\left(\Pi_{k}(t, x), \Pi_{k}(t, y)\right) p_{i k}(y) a e^{-a t} d t .
\end{aligned}
$$

Without any loss of generality we may assume that $|x|_{*} \leq|y|_{*}$. For $k \geq 2$, we have

$$
\varrho\left(\Pi_{k}(t, x), \Pi_{k}(t, y)\right) \leq r_{k}\left(|y|_{*}\right) e^{-\lambda t} \varrho(x, y)
$$

and

$$
p_{i k}(y)=\bar{q}_{k}\left(|y|_{*}\right) \leq q_{k}\left(|y|_{*}\right) \leq \frac{\delta}{1+r_{k}\left(|y|_{*}\right)} .
$$

Thus

$$
\begin{aligned}
\sum_{k=2}^{N} p_{i k}(y) \varrho\left(\Pi_{k}(t, x), \Pi_{k}(t, y)\right) & \leq \sum_{k=2}^{N} \frac{\delta}{1+r_{k}\left(|y|_{*}\right)} r_{k}\left(|y|_{*}\right) e^{-\lambda t} \varrho(x, y) \\
& \leq \sum_{k=2}^{N} \delta e^{-\lambda t} \varrho(x, y)=(N-1) \delta e^{-\lambda t} \varrho(x, y) .
\end{aligned}
$$

Moreover

$$
p_{i 1}(y) \varrho\left(\Pi_{1}(t, x), \Pi_{1}(t, y)\right) \leq p_{i 1}(y) L_{1} e^{-\lambda t} \varrho(x, y),
$$

thus

$$
\begin{aligned}
\sum_{k=1}^{N} p_{i k}(y) \varrho\left(\Pi_{k}(t, x), \Pi_{k}(t, y)\right) & \leq\left(p_{i 1}(y) L_{1}+(N-1) \delta\right) e^{-\lambda t} \varrho(x, y) \\
& \leq\left(L_{1}+(N-1) \delta\right) e^{-\lambda t} \varrho(x, y) \\
& \leq L e^{-\lambda t} \varrho(x, y)
\end{aligned}
$$

where $L=1+\lambda / a-\left(1+\lambda / a-L_{1}\right) / N$.

From (3.5) it now follows that

$$
\begin{aligned}
\mid U f(x, i)-U f & (y, i) \mid \\
& \leq \sum_{k=1}^{N}\left|p_{i k}(x)-p_{i k}(y)\right|+K \int_{0}^{\infty} L a e^{-(a+\lambda) t} \varrho(x, y) d t \\
& \leq \sum_{k=1}^{N}\left|p_{i k}(x)-p_{i k}(y)\right|+K r \varrho(x, y)
\end{aligned}
$$

where $r=a L /(a+\lambda)$. Moreover from Lemma 2.3 we obtain

$$
\left|p_{i k}(x)-p_{i k}(y)\right|=\left|\bar{q}_{k}\left(|x|_{*}\right)-\bar{q}_{k}\left(|y|_{*}\right)\right| \leq \varepsilon \varrho(x, y) \quad \text { for } k=2, \ldots, N .
$$


Thus

$$
\begin{aligned}
\sum_{k=1}^{N}\left|p_{i k}(x)-p_{i k}(y)\right| & \leq\left|p_{i 1}(x)-p_{i 1}(y)\right|+\sum_{k=2}^{N}\left|p_{i k}(x)-p_{i k}(y)\right| \\
& \leq 2 \sum_{k=2}^{N}\left|\bar{q}_{k}\left(|x|_{*}\right)-\bar{q}_{k}\left(|y|_{*}\right)\right| \leq 2(N-1) \varepsilon \varrho(x, y) .
\end{aligned}
$$

From (3.6) we finally obtain

$$
|U f(x, i)-U f(y, i)| \leq 2(N-1) \varepsilon \varrho(x, y)+K r \varrho(x, y),
$$

which reduces to

$$
|U f(x, i)-U f(y, i)| \leq K \varrho(x, y)
$$

by the definition of $K$, and completes the proof of the nonexpansiveness.

Claim II. The operator $P$ is semi-concentrating.

Fix $\gamma>0$. Consider the function

$$
V(x, k)=\varrho\left(x, x_{*}\right) \quad \text { for } x \in Y \text { and } k=1, \ldots, N \text {. }
$$

By (2.3) and the definition of $V, p_{i k}$ and $\sigma_{i}$ we have

$$
\begin{aligned}
U V(x, k)= & \sum_{s=1}^{N} \int_{0}^{\infty} \varrho\left(\Pi_{s}(t, x), x_{*}\right) a e^{-a t} p_{k s}(x) d t \\
\leq & \int_{0}^{\infty} \varrho\left(\Pi_{1}(t, x), x_{*}\right) a e^{-a t} p_{k 1}(x) d t+\sum_{s=2}^{N} \sigma_{s}\left(|x|_{*}\right) \frac{\delta}{1+\sigma_{s}\left(|x|_{*}\right)} \\
\leq & \int_{0}^{\infty} \varrho\left(\Pi_{1}(t, x), \Pi_{1}\left(t, x_{*}\right)\right) a e^{-a t} p_{k 1}(x) d t \\
& +\int_{0}^{\infty} \varrho\left(\Pi_{1}\left(t, x_{*}\right), x_{*}\right) a e^{-a t} p_{k 1}(x) d t+(N-1) \delta \\
\leq & L_{1} \frac{a}{\lambda+a} \varrho\left(x, x_{*}\right)+M+(N-1) \delta
\end{aligned}
$$

where

$$
M=\max _{1 \leq k \leq N} \sup _{t \geq 0} \varrho\left(\Pi_{k}\left(t, x_{*}\right), x_{*}\right) .
$$

Setting $b=M+(N-1) \delta$ and $\beta=L_{1} a /(\lambda+a)$, we have

$$
U V(x, k) \leq \beta V(x, k)+b .
$$


Now define

$$
m_{n}=\left\langle V, \bar{\mu}_{n}\right\rangle, \quad n=0,1, \ldots
$$

Consider first the case $m_{0}<\infty$. Using the recurrence formula $\bar{\mu}_{n+1}=P \bar{\mu}_{n}$ and (3.7) we have

$$
m_{n+1}=\left\langle V, P \bar{\mu}_{n}\right\rangle=\left\langle U V, \bar{\mu}_{n}\right\rangle \leq\left\langle\beta V+b, \bar{\mu}_{n}\right\rangle=\beta m_{n}+b .
$$

By an induction argument this gives

$$
m_{n+1} \leq \beta^{n} m_{0}+\frac{b}{1-\beta} .
$$

Define

$$
R=\frac{2 b}{\gamma(1-\beta)} .
$$

Using the Chebyshev inequality we get

$$
P^{n} \bar{\mu}_{0}(B)=\bar{\mu}_{n}(B) \geq 1-\gamma \quad \text { for } n \geq n_{0} \text { and } \bar{\mu}_{0} \in \mathcal{M}_{1}(\bar{Y})
$$

where $B=K\left(x_{*}, R\right) \times\{1, \ldots, N\}$. The general case $m_{0} \leq \infty$ can be reduced to the previous one as follows. For given $\delta>0$ we choose a bounded Borel set $A \subset \bar{Y}$ such that $\bar{\mu}_{0}(A) \geq 1-\delta$. Setting

$$
\nu_{0}(D)=\frac{\bar{\mu}_{0}(A \cap D)}{\bar{\mu}_{0}(A)}
$$

we define a probability measure $\nu_{0}$ supported on $A$ for which the initial moment $\bar{m}_{0}=\left\langle V, \nu_{0}\right\rangle$ is finite. Thus

$$
P^{n} \nu_{0}(B) \geq 1-\gamma \quad \text { for } n \geq n_{0} .
$$

Since $\bar{\mu}_{0}(D) \geq \bar{\mu}_{0}(D \cap A)$, we have

$$
P^{n} \bar{\mu}_{0}(B) \geq \bar{\mu}_{0}(A) P^{n} \nu_{0}(B) \geq(1-\delta)(1-\gamma) .
$$

Choosing $\delta$ sufficiently small we obtain

$$
P^{n} \bar{\mu}_{0}(B) \geq 1-\bar{\gamma} \quad \text { for } n \geq n_{0} .
$$

Now we define the families of functions $\Pi_{k_{n} \ldots k_{1}}^{t_{n} \ldots t_{1}}: Y \rightarrow Y$ and $\bar{\Pi}_{k_{n} \ldots k_{1}}^{t_{n} \ldots t_{1}}$ : $\bar{Y} \rightarrow \bar{Y}\left(t_{i} \in \mathbb{R}_{+}, k_{i} \in\{1, \ldots, N\}\right.$ for $\left.i=1, \ldots, n\right)$ by the recurrence relations

$$
\begin{aligned}
\Pi_{k_{1}}^{t_{1}}(y) & =\Pi_{k_{1}}\left(t_{1}, y\right) \\
\Pi_{k_{n} \ldots k_{1}}^{t_{n} \ldots t_{1}}(y) & =\Pi_{k_{n}}\left(t_{n}, \Pi_{k_{n-1} \ldots k_{1}}^{t_{n-1} \ldots t_{1}}(y)\right) \quad \text { for } y \in Y
\end{aligned}
$$

and

$$
\begin{aligned}
\bar{\Pi}_{k_{1}}^{t_{1}}(y, s) & =\left(\Pi_{k_{1}}^{t_{1}}(y), k_{1}\right), \\
\bar{\Pi}_{k_{n} \ldots k_{1}}^{t_{n} \ldots t_{1}}(y, s) & =\left(\Pi_{k_{n} \ldots k_{1}}^{t_{n} \ldots t_{1}}(y), k_{n}\right) \quad \text { for }(y, s) \in \bar{Y} .
\end{aligned}
$$


Using equation (2.3) $n$ times, we obtain

$$
\begin{aligned}
& U^{n} f(y, i) \\
& =\sum_{k_{1}, \ldots, k_{n}} \underbrace{\int_{\mathbb{R}_{+}} \ldots \int_{\mathbb{R}_{+}}}_{n} p_{i k_{1}}(y) p_{k_{1} k_{2}}\left(\Pi_{k_{1}}^{t_{1}}(y)\right) \ldots p_{k_{n-1} k_{n}}\left(\Pi_{k_{n-1} \ldots k_{1}}^{t_{n-1} \ldots t_{1}}(y)\right) \\
& \quad \times f\left(\bar{\Pi}_{k_{n} \ldots k_{1}}^{t_{n} \ldots t_{1}}(y, i)\right) a^{n} e^{-a\left(t_{1}+\ldots+t_{n}\right)} d t_{1} \ldots d t_{n} .
\end{aligned}
$$

By (3.9) there exists a bounded Borel set $F \subset \bar{Y}$ such that for every $\mu \in$ $\mathcal{M}_{1}(\bar{Y})$ there exists an integer $n_{1}=n_{1}(\mu)$ for which

$$
P^{n} \mu(F) \geq 1 / 2 \quad \text { for } n \geq n_{1} \text {. }
$$

Let $\bar{t}>0$ be such that

$$
r_{0}=\sup _{1 \leq k \leq N} L_{k} e^{-\lambda \bar{t}}<1
$$

Fix $\alpha>0$. We can find an integer $m$ such that

$$
r_{0}^{m} \operatorname{diam}_{\bar{\varrho}} F \leq \alpha / 2
$$

Fix $(y, s) \in F$ and set

$$
C=\bigcup_{j_{1}, \ldots, j_{m}=1}^{N} K\left(\bar{\Pi}_{j_{m} \ldots j_{1}}^{\bar{t} \ldots \bar{t}}(y, s), \alpha\right) .
$$

Obviously $C \in \mathcal{C}_{\alpha}$. By continuity there exists a constant $\tau, 0<\tau<\bar{t}$, such that

$$
\bar{\varrho}\left(\bar{\Pi}_{j_{m} \ldots j_{1}}^{\bar{t} \ldots \bar{t}}(y, s), \bar{\Pi}_{j_{m} \ldots j_{1}}^{t_{m} \ldots t_{1}}(y, s)\right)<\alpha / 2
$$

for all sequences $\left(j_{1}, \ldots, j_{m}\right) \in\{1, \ldots, N\}^{m}$ and $t_{1}, \ldots, t_{m} \in[\bar{t}, \bar{t}+\tau]$. Set

$$
\sigma=\inf \left\{p_{i j}(x): x \in Y, i, j \in\{1, \ldots, N\}\right\}
$$

From Lemma 2.3 it follows that $\sigma>0$.

We now prove that

$$
\liminf _{n \rightarrow \infty} P^{n} \mu(C) \geq \frac{1}{2}\left(\frac{\sigma}{N} \tau a e^{-a(\bar{t}+\tau)}\right)^{m}
$$

for all $\mu \in \mathcal{M}_{1}(\bar{Y})$. To do this fix $\mu \in \mathcal{M}_{1}(\bar{Y})$. There exists an integer $n_{1}=n_{1}(\mu)$ for which (3.11) holds. Let $n=\bar{n}+m$ for some $\bar{n} \geq n_{1}$. Using (3.10) we get 
(3.16) $\quad P^{n} \mu(C)$

$$
\begin{aligned}
= & \int_{\bar{Y}} \sum_{k_{1}, \ldots, k_{m}} \underbrace{\int_{\mathbb{R}_{+}} \ldots \int_{\mathbb{R}_{+}}}_{m} p_{s k_{1}}(w) p_{k_{1} k_{2}}\left(\Pi_{k_{1}}^{t_{1}}(w)\right) \ldots p_{k_{m-1} k_{m}}\left(\Pi_{k_{m-1} \ldots k_{1}}^{t_{m-1} \ldots t_{1}}(w)\right) \\
& \times \mathbf{1}_{C}\left(\bar{\Pi}_{k_{m} \ldots k_{1}}^{t_{m} \ldots t_{1}}(w, r)\right) a^{m} e^{-a\left(t_{1}+\ldots+t_{m}\right)} d t_{1} \ldots d t_{m} d P^{\bar{n}} \mu(w, r) .
\end{aligned}
$$

Consider the space

$$
Z=F \times \underbrace{[\bar{t}, \bar{t}+\tau] \times \ldots \times[\bar{t}, \bar{t}+\tau]}_{m \text { times }}
$$
with the product measure $P^{\bar{n}} \mu \otimes \underbrace{l_{1} \otimes \ldots \otimes l_{1}}_{m \text { times }}$, where $l_{1}$ denotes the Lebesgue
measure. Define

$$
\begin{aligned}
Z_{j_{1} \ldots j_{m}}=\{((w, r) & \left., t_{1}, \ldots, t_{m}\right) \in Z: \\
& \left.\bar{\varrho}\left(\bar{\Pi}_{j_{m} \ldots j_{1}}^{t_{m} \ldots t_{1}}(y, s), \bar{\Pi}_{j_{m} \ldots j_{1}}^{t_{m} \ldots t_{1}}(w, r)\right) \leq r_{0}^{m} \bar{\varrho}((w, r),(y, s))\right\}
\end{aligned}
$$

for $j_{1}, \ldots, j_{m} \in\{1, \ldots, N\}$. Applying (3.1) ( $m$ times) we see that for every $(w, r) \in F$ and $\left(t_{1}, \ldots, t_{m}\right) \in[\bar{t}, \bar{t}+\tau]$ there exists a sequence $\left(i_{1}, \ldots, i_{m}\right) \in$ $\{1, \ldots, N\}^{m}$ such that $\left((w, r), t_{1}, \ldots, t_{m}\right) \in Z_{i_{1} \ldots i_{m}}$.

Hence we deduce that

$$
Z=\bigcup_{j_{1}, \ldots, j_{m}=1}^{N} Z_{j_{1} \ldots j_{m}}
$$

Since

$$
\left(P^{\bar{n}} \mu \otimes l_{1} \otimes \ldots \otimes l_{1}\right)(Z) \geq \frac{1}{2} \tau^{m}
$$

there exists a sequence $\left(k_{1}, \ldots, k_{m}\right) \in\{1, \ldots, N\}^{m}$ such that

$$
\left(P^{\bar{n}} \mu \otimes l_{1} \otimes \ldots \otimes l_{1}\right)\left(Z_{k_{1} \ldots k_{m}}\right) \geq \frac{\tau^{m}}{2 N^{m}} .
$$

Combining (3.13) and (3.14) with (3.16) and (3.18) we obtain

$$
\begin{aligned}
P^{n} \mu(C) \geq & \sigma^{m} \int_{Z_{k_{1} \ldots k_{m}}} \ldots \int \mathbf{1}_{C}\left(\bar{\Pi}_{k_{m} \ldots k_{1}}^{t_{m} \ldots t_{1}}(w, r)\right) \\
& \times a^{m} e^{-a\left(t_{1}+\ldots+t_{m}\right)} d t_{1} \ldots d t_{m} d P^{\bar{n}}(w, r) \\
\geq & \frac{1}{2}\left[\frac{\sigma}{N} \tau a e^{-a(\bar{t}+\tau)}\right]^{m},
\end{aligned}
$$

which finishes the proof of Claim II.

From Lemma 2.1(i) it follows that the operator $P$ given by (2.4) has an invariant measure.

CLAIM III. The operator $P$ satisfies a lower bound condition, namely, for every $\beta>0$ there is $\Delta>0$ such that for any $\mu_{1}, \mu_{2} \in \mathcal{M}_{1}(\bar{Y})$ there exists a 
Borel measurable set $A$ with $\operatorname{diam}_{\bar{\varrho}} A \leq \beta$ and an integer $n_{0}$ for which

$$
P^{n_{0}} \mu_{k}(A) \geq \Delta \quad \text { for } k=1,2 .
$$

Fix $\beta>0$. Since $P$ is semi-concentrating, Lemma 2.1 shows that there exists a compact set $H \subset \bar{Y}$ such that

$$
\mu(H) \geq 4 / 5 \quad \text { for } \mu \in \Gamma \text {. }
$$

Now let $p \in \mathbb{N}$ be such that

$$
r_{0}^{p} \operatorname{diam}_{\bar{\varrho}} H \leq \beta / 3,
$$

where $r_{0}$ is defined by (3.12). For $(y, s) \in H$ and $\left(k_{1}, \ldots, k_{p}\right) \in\{1, \ldots, N\}^{p}$ we define the open sets

$$
\begin{aligned}
O_{(y, s)}= & \bigcap_{k_{1}, \ldots, k_{p}=1}^{N}\{(w, r) \in \bar{Y}: \\
& \left.\bar{\Pi}_{k_{p} \ldots k_{1}}^{\bar{t} \ldots \bar{t}}((w, r)) \in K\left(\bar{\Pi}_{k_{p} \ldots k_{1}}^{\bar{t} \ldots \bar{t}}(y, s), \beta / 3\right)\right\} .
\end{aligned}
$$

Take $\left(y_{1}, s_{1}\right), \ldots,\left(y_{h}, s_{h}\right) \in H$ such that

$$
H \subset \bigcup_{i=1}^{h} O_{\left(y_{i}, s_{i}\right)}
$$

and define

$$
G=\bigcup_{i=1}^{h} O_{\left(y_{i}, s_{i}\right)} .
$$

By continuity there exists $\bar{\gamma}>0$ such that for all $(y, s) \in H$ and all sequences $\left(k_{1}, \ldots, k_{p}\right) \in\{1, \ldots, N\}^{p}$ we have

$$
\bar{\varrho}\left(\bar{\Pi}_{k_{p} \ldots k_{1}}^{t_{p} \ldots t_{1}}(y, s), \bar{\Pi}_{k_{p} \ldots k_{1}}^{\bar{t} \ldots \bar{t}}(y, s)\right)<\beta / 6
$$

for $t_{i} \in[\bar{t}, \bar{t}+\bar{\gamma}], i=1, \ldots, p$.

We show that (3.20) holds with

$$
\Delta=\frac{1}{2 h}\left[\sigma \bar{\gamma} a e^{-a(\bar{t}+\bar{\gamma})}\right]^{p} .
$$

Fix $\mu_{1}, \mu_{2} \in \mathcal{M}_{1}(\bar{Y})$. Set $\bar{\mu}=\left(\mu_{1}+\mu_{2}\right) / 2$. Lemma 2.1 gives $\omega(\bar{\mu}) \neq \emptyset$. Let $\left\{n_{j}\right\}_{j \geq 1}$ be a sequence of integers such that $\left\{P^{n_{j}} \bar{\mu}\right\}_{j \geq 1}$ converges to some measure $\mu_{*} \in \mathcal{M}_{1}(\bar{Y})$. By the Alexandrov theorem we have

$$
\liminf _{j \rightarrow \infty} P^{n_{j}} \bar{\mu}(G) \geq \mu_{*}(G) \geq \mu_{*}(H) \geq 4 / 5 .
$$

Hence there exists $n_{0} \in \mathbb{N}$ such that

$$
P^{n_{0}} \bar{\mu}(G) \geq 3 / 4
$$


and consequently

$$
P^{n_{0}} \mu_{i}(G) \geq 1 / 2 \quad \text { for } i=1,2 \text {. }
$$

Thus we get

$$
P^{n_{0}} \mu_{1}\left(O_{\left(y_{l}, s_{l}\right)}\right) \geq \frac{1}{2 h} \quad \text { and } \quad P^{n_{0}} \mu_{2}\left(O_{\left(y_{k}, s_{k}\right)}\right) \geq \frac{1}{2 h}
$$

for some $l, k \in\{1, \ldots, h\}$. Write for simplicity $O_{1}=O_{\left(y_{l}, s_{l}\right)}, O_{2}=O_{\left(y_{k}, s_{k}\right)}$. Now, (3.1) and (3.22) imply

$$
\bar{\varrho}\left(\bar{\Pi}_{i_{p} \ldots i_{1}}^{\bar{t} \ldots \bar{t}}\left(y_{l}, s_{l}\right), \bar{\Pi}_{i_{p} \ldots i_{1}}^{\bar{t} \ldots \bar{t}}\left(y_{k}, s_{k}\right)\right) \leq r_{0}^{p} \operatorname{diam}_{\bar{\varrho}} H \leq \beta / 3
$$

for some $\left(i_{p}, \ldots, i_{1}\right) \in\{1, \ldots, N\}^{p}$.

Define $A=A_{1} \cup A_{2}$, where

$$
A_{i}=\operatorname{cl}\left\{\bar{\Pi}_{i_{p} \ldots i_{1}}^{t_{p} \ldots t_{1}}(y, s):(y, s) \in O_{i}, t_{j} \in[\bar{t}, \bar{t}+\bar{\gamma}] \text { for } j \in\{1, \ldots, p\}\right\}
$$

for $i=1,2$ (here cl denotes closure in the space $\bar{Y}$ ). Using (3.23) and (3.26) we check at once that $\operatorname{diam}_{\bar{\varrho}} A \leq \varepsilon$. Proceeding analogously to the proof of the estimates (3.16) and (3.19) we get

$$
P^{n_{0}+p} \mu_{i}(A) \geq \frac{1}{2 h}\left[\sigma \bar{\gamma} a e^{-a(\bar{t}+\bar{\gamma})}\right]^{p} \quad \text { for } i=1,2,
$$

which finishes the proof of Claim III.

CLAIM IV. The sequence $\left\{\mu_{n}\right\}_{n \geq 1}$ defined by (2.2) is weakly convergent to a distribution $\mu_{*}$.

Since by Claim II the operator $P$ given by (2.4) has an invariant measure, Lemma 2.2 yields

$$
\lim _{n \rightarrow \infty}\left\|P^{n} \mu_{1}-P^{n} \mu_{2}\right\|_{\mathcal{F}}=0 \quad \text { for } \mu_{1}, \mu_{2} \in \mathcal{M}_{1}(\bar{Y})
$$

Thus the operator $P$ is asymptotically stable. Hence there exists an invariant measure $\bar{\mu}_{*}$ such that

$$
\lim _{n \rightarrow \infty}\left\langle\bar{f}, \bar{\mu}_{n}\right\rangle=\left\langle\bar{f}, \bar{\mu}_{*}\right\rangle \quad \text { for } \bar{f} \in C(\bar{Y}),
$$

where $\bar{\mu}_{n+1}=P \bar{\mu}_{n}$. Hence

$$
\lim _{n \rightarrow \infty} \int_{\bar{Y}} \bar{f}(x, i) d \bar{\mu}_{n}(x, i)=\int_{\bar{Y}} \bar{f}(x, i) d \bar{\mu}_{*}(x, i) \quad \text { for } \bar{f} \in C(\bar{Y}) .
$$

Further, for every $f \in C(Y)$ we define the sequence of functions $\bar{f}_{j}: \bar{Y} \rightarrow \bar{Y}$, $j=1, \ldots, N$, by the formula

$$
\bar{f}_{j}(x, i)= \begin{cases}f(x) & \text { for } i=j \\ 0 & \text { for } i \neq j\end{cases}
$$


It is evident that each $\bar{f}_{j}$ belongs to $C(\bar{Y})$. From (3.29) it follows that

$$
\lim _{n \rightarrow \infty} \sum_{j=1}^{N} \int_{\bar{Y}} \bar{f}_{j}(x, i) d \bar{\mu}_{n}(x, i)=\sum_{j=1}^{N} \int_{\bar{Y}} \bar{f}_{j}(x, i) d \bar{\mu}_{*}(x, i),
$$

and consequently

$$
\lim _{n \rightarrow \infty} \sum_{j=1}^{N} \int_{Y} f(x) \bar{\mu}_{n}(d x \times\{j\})=\sum_{j=1}^{N} \int_{Y} f(x) \bar{\mu}_{*}(d x \times\{j\}) .
$$

Setting

$$
\mu_{*}(A)=\sum_{j=1}^{N} \bar{\mu}_{*}(A \times\{j\}) \quad \text { for } A \in \mathcal{B}(Y)
$$

and using the definitions of $\mu_{n}$ and $\bar{\mu}_{n}$ we finally obtain

$$
\lim _{n \rightarrow \infty} \int_{Y} f(x) \mu_{n}(d x)=\int_{Y} f(x) \mu_{*}(d x) \quad \text { for } f \in C(Y) .
$$

\section{References}

[1] R. Fortet et B. Mourier, Convergence de la répartition empirique vers la répartition théorétique, Ann. Sci. École Norm. Sup. 70 (1953), 267-285.

[2] K. Horbacz, Randomly connected dynamical systems-asymptotic stability, Ann. Polon. Math. 68 (1998), 31-50.

[3] A. Lasota, From fractals to stochastic differential equations, in: Chaos-the Interplay between Stochastic and Deterministic Behaviour (Karpacz'95), Springer, 1995, 235255.

[4] A. Lasota and J. Myjak, Semifractals on Polish spaces, Bull. Polish Acad. Sci. Math. 46 (1998), 179-196.

[5] A. Lasota and J. A. Yorke, Lower bound technique for Markov operators and iterated function systems, Random Comput. Dynam. 2 (1994), 41-77.

[6] T. Szarek, Markov operators acting on Polish spaces, Ann. Polon. Math. 67 (1997), 247-257.

Institute of Mathematics

Silesian University

Bankowa 14

40-007 Katowice, Poland

E-mail: horbacz@ux2.math.us.edu.pl

Reçu par la Rédaction le 5.2.2000

Révisé le 28.6.2000 